\title{
Designing Interactive Blimps as Puppets
}

\author{
Hideki Yoshimoto ${ }^{1}$, Kazuhiro $\mathrm{Jo}^{2}$, and Koichi Hori ${ }^{1}$ \\ ${ }^{1}$ Department of Aeronautics and Astronautics, University of Tokyo \\ yoshimoto@ailab.t.u-tokyo.ac.jp \\ ${ }^{2}$ Culture Lab, Newcastle University
}

\begin{abstract}
In this paper we propose four models of unmanned blimps: Robots, Pets, Agents, and Puppets, according to whether they are autonomous or not and whether they are shown to people or not. Robots and Pets are autonomous and Agents and Puppets are not autonomous. Robots and Agents are shown to people and Pets and Puppets are not shown to people. Based on these models, we approach toward interactive blimps as puppets, which visualize performances from people to people with real time effects and motions. We implemented prototype applications where people could make performances through controls of the blimp's light effects and flight motions with voice via mobile phones and a physical controller. We organized observations of these prototypes at a laboratory experiment and demo exhibitions. We also discuss our models based on spectators' experience.
\end{abstract}

Keywords: Blimp, Airship, Performance, Interaction, Art, Installation.

\section{Introduction}

Unmanned blimps have been utilized in various fields such as hobbies, arts $[1,5,7,14]$, science [11], and mass communications [8, 9]. Aerial Do-It-Yourself (DIY) projects are also spreading [2], which would make unmanned blimps more familiar and diversified. Blimps have following features compared with other aerial vehicles. First, blimps could fly at slow speed. Second, blimps are soft and collision between small blimps and people is not so dangerous. Third, the envelopes could be customized as canvas. These features make it easy to utilize blimps in various ways in daily life.

In this paper we propose four models of unmanned blimps: Robots, Pets, Agents, and Puppets, according to whether they are autonomous or not and whether they are shown to people or not. Based on these models, we approach toward interactive blimps as puppets, which visualize performances from people to people with real time effects and motions.

\section{Robots, Pets, Agents, Puppets}

We propose four models of unmanned blimps: Robots, Pets, Agents, and Puppets. We first divide unmanned blimps into two groups according to whether they are autonomous 
Table 1. Robots, Pets, Agents, Puppets

\begin{tabular}{|c|c|c|c|}
\hline \multicolumn{4}{|c|}{ Unmanned Blimps } \\
\hline Autonomous & \multicolumn{2}{c|}{ Not Autonomous } \\
\hline Not Shown to People & Shown to People & Not Shown to People & Shown to People \\
\hline ROBOTS & PETS & AGENTS & PUPPETS \\
\hline & & Operators & \\
\hline & & & \\
& & & \\
& & & \\
\hline
\end{tabular}

or not. Then we propose two models for each group according to whether they are shown to people or not. In these models, we define two roles of people: Operators, who pilot and control the blimps, and Spectators, who observe the blimps.

Robots. Robots are blimps that are autonomous and not shown to people. The blimps do their own jobs by themselves like industrial robots.

Blimps used as antennas or weather observers are classified as robots.

Pets. Pets are blimps that are autonomous and shown to people. Spectators observe and enjoy the blimps' flight motions as they enjoy seeing animals.

Autonomous Light Air Vessels (ALAVs) is a project where blimps are designed to flock each other or to roam for lights [1]. Blubber Bot is a DIY kit version of the ALAVs. Alan Kay's blimp in Vivarium project flies autonomously following a flashlight. Kawamura's entertainment blimp is controlled to hover and make several motions triggered by people's handclaps [6]. Ardublimp and Blimpduino are DIY autonomous blimps that fly around the ground beacons [2]. These blimps are classified as pets.

Agents. Agents are blimps that are not autonomous and not shown to people. Operators control the blimps for their own purposes as business clients have agencies execute their own jobs. Spectators do not concern with these activities.

"PRoP: Personal Roving Presence" by Paulos and Canny is a project where people remotely control blimps equipped with cameras and microphones through Internet to feel as if they were on board [11]. Floating Eye is an installation where people wearing head-mounted displays see wide-angle images captured by the blimp floating in the air [5]. "Naked Bandit/here, not here/white sovereign" is Knowbotic Research's installation where people control a blimp using a physical controller to attack targets with it [7]. Radio-Controlled (RC) toy blimps are controlled by operators and their purposes are the controls itself. These blimps are classified as agents.

Puppets. Puppets are blimps that are not autonomous and shown to people. Operators control the blimps to make performances to spectators as puppeteers animate puppets 
to make performances to spectators. Additional visual effects (e.g., light illuminations, graphic images, and videos) would play important roles for the performances.

Advertising or broadcasting blimps (e.g., Lightship [8]) are classified as puppets in that they are piloted and visualize representations from clients to customers. However, these representations with static images or programmed videos do not react to operators in real time. We approach toward interactive blimps as puppets that visualize performances reacting to operators in real time.

\section{Designing Interactive Blimps as Puppets through Prototyping}

As examples of interactive blimps as puppets, we implemented two prototype applications. One is participative performance, where the public control and observe the blimps. The other is show performance, where a skilled operator controls the blimp in front of spectators. This section explains our platform system and these applications. We also describe the observations of them.

Platform System. Our platform system consists of a blimp, wireless communication modems, and OSC-Serial Translator. We built indoor blimps equipped with three propeller motors (two for horizontal movement and one for vertical movement) and Light-Emitting Diodes (LEDs), which realize 3D motions and full-color light effects. We implemented fuzzy control systems for stabile flight motions [10]. The latest blimp is $1.15 \mathrm{~m}$ long and the total weight is $220 \mathrm{~g}$. We utilized Arduino as the micro controller on board and XBee as the wireless communication modems. We employed Open Sound Control (OSC) protocol for internal communication between the platform system and applications' interfaces. OSC-Serial Translator translates the OSC messages into serial messages to communicate with the blimp.

Participative Performance. In participative performance the public control and observe the blimps. In our prototype application, they control the blimp's motions and

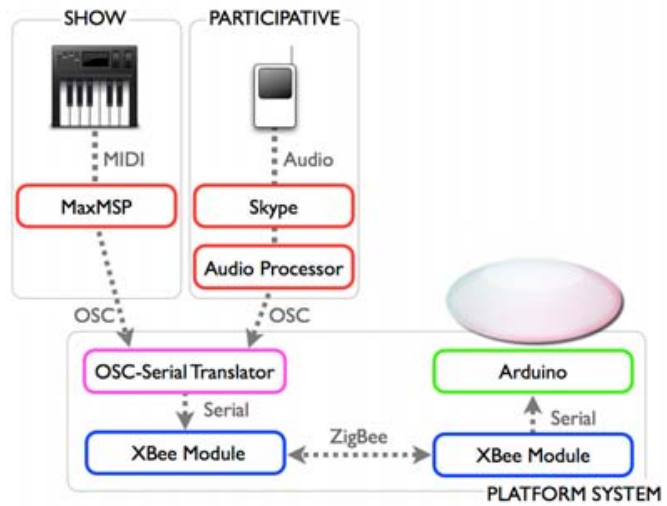

Fig. 1. Configuration

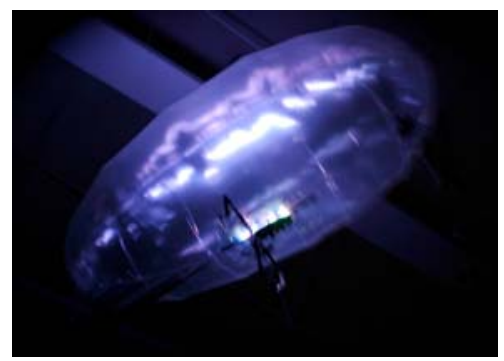

Fig. 2. Blimp 
light effects with voice via mobile phones. Voice via mobile phones is advantageous as an interface for the public in that it does not require special preparations. It also enables them to control and watch the blimp simultaneously. We employed volume, pitch transitions [4], filled pauses (i.e., continuous voice like "Ahhh") [3] and word recognition as the vocal interfaces. Audio Processor analyzes incoming voice from mobile phones via Skype. The horizontal movement is controlled with combination of word recognition and the filled pauses. For example, people say, "Go, Ahhh", then the blimp goes straight during "Ahhh". The vertical movement (up/down) represents the pitch transitions of the voice (upward/downward). Brightness of the LEDs represents the volume of the voice. Color of the LEDs represents the pitch transitions. When the pitch of the voice is going up/down, the light tinges with green/red.

Show Performance. In show performance a skilled operator controls the blimp in front of spectators. In our prototype application, the operator control the blimp's motions and light effects with a physical controller. A physical controller is advantageous for complex controls such as those to music or dance because of the ability in numerical and prompt manipulations. We utilized sliders and buttons of a MIDI keyboard as the physical controller. Max/MSP translates the MIDI messages into OSC messages. The horizontal flight modes such as "Go Straight", "Spin", or "Right", and the vertical movement are switched with buttons. Brightness of the each color was controlled with sliders. Automatic light effects such as flash and oscillation are also programmed and switched with buttons.

Observations. We organized a laboratory experiment in a dim high-ceiling room. For observation of the participative performance application, two persons participated with their mobile phones. The vertical movement and the light effects well represented the participants' vocal controls. However, the participants had difficulties in controlling the blimp's horizontal movement with word commands because the voice through the system included noises and latency. For observation of the show performance application, one of the authors made a musical performance with the blimp. Kinds of motions such as circular flight, slow swing, and hovering and fullcolor light effects could represent the musical tunes. We also introduced our participative performance application in demo exhibitions at Make: Tokyo Meeting 02 (MTM 02, http://www.oreilly.co.jp/mtm/02/) and the Workshop on Media Arts, Science, and Technology (MAST) [13]. In MTM 02, more than 120 persons including children experienced controls of only light effects using voice. It was easy to learn and enjoyable for both adults and children. In MAST, more than 25 persons experienced the controls of the flight motions and light effects using voice. They enjoyed the exhibition while they had difficulties in controlling vertical and horizontal movements in balance. Light effects represented the volume and the pitch transitions. Through these observations, we recognized that verbal interfaces are not suitable for flight motions, which requires controls of multiple parameters. In both of flight motions and light effects, simpler effects work well in the participative performance and more complex effects work well in the show performance. 


\section{Discussion}

In this section, we would like to discuss the differences between our four models based on the taxonomy proposed by Reeves et al [12]. The goal of this taxonomy is designing the spectators' experience according to how they hide or reveal manipulations and effects in interaction. Manipulations are the actions carried out by the performers (operators). Effects are the results of these manipulations. Figure 3 is the classification of unmanned blimps mentioned in the former sections.

Blimps as puppets are characterized with amplified visual effects. Manipulations in our participative performance are revealed because the public behave as both operators and spectators. Manipulations in our show performance are partially hidden because the spectators could see only rough motions of the operators. Manipulations in Lightship and Advertising Blimp are hidden because the spectators could not see the advertisers or broadcasters.

Blimps as pets are characterized with revealed effects, which means that spectators observe the blimps' flight itself but no amplified effects, and hidden or partially hidden manipulations caused by autonomous operations. Manipulations in Vivarium, Kawamura's Blimp, ALAV, and Blubber Bot are partially revealed because they operate autonomously but the spectators could affect them. Manipulations in Ardublimp and Blimpduino are hidden because they could not be affected by spectators.

Blimps as agents are characterized with transformed or hidden effects. In Toy RC Blimp and Naked Bandit, effects are transformed because the spectators could observe the blimps and the operators but they could not experience the controls that the operators experience. Manipulations of these projects are partially hidden because spectators could not see details of the controls. In Floating Eye and PRoP, effects are hidden because the spectators could not see the images that operators see. Manipulations in Floating Eye are revealed because the spectators could observe the controls of the operators. Manipulations in PRoP are hidden because the operators control the blimp remotely in distant places.

Blimps as robots are characterized with hidden effects and hidden manipulations. It is because the spectators could not see the blimps themselves.

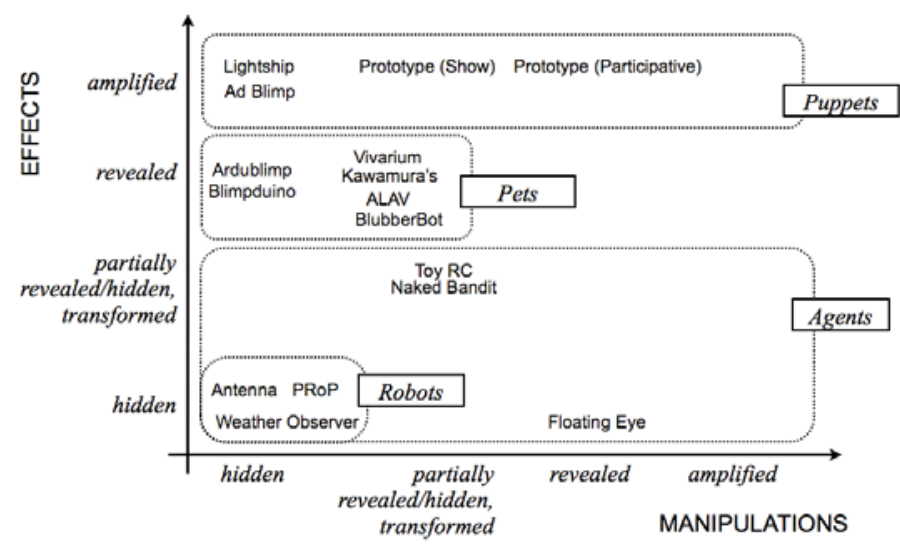

Fig. 3. Classification based on Spectators' Experience 


\section{Conclusion and Future Work}

In this paper we proposed four models of unmanned blimps: Robots, Pets, Agents, and Puppets, and described our approach toward interactive blimps as puppets. Interactive blimps as puppets visualize operators' performances to spectators with real time effects and motions. As examples of interactive blimps as puppets, we implemented two prototype applications: participative performance and show performance. In the former, the public control the blimp with voice via mobile phones. In the latter, a skilled operator controls the blimp with a physical controller. In both applications the blimp visualize the performances with its full-color light effects and 3D motions. Through observations of the prototypes in a laboratory experiment and demo exhibitions, we recognized that simpler effects work well in the participative performance and more complex effects work well in the show performance. Our next interests are in interaction between multiple blimps and people. Multiple blimps could not only interact with each participant but also illuminate and direct the whole space. This kind of interaction would produce new performances in the air.

\section{References}

1. Berk, J., Mitter, N.: Autonomous Light Air Vessels (ALAVs). In: ACM MULTIMEDIA 2006, pp. 1029-1030. ACM Press, New York (2006)

2. DIY Drones, http: / / diydrones.com/

3. Goto, M., Itou, K., Hayamizu, S.: A Real-Time System Detecting Filled Pauses in Spontaneous Speech. IEICE J83-D-II, 11, 2330-2340 (2000)

4. Igarashi, T., Hughes, J.: Voice as Sound: Using Nonverbal Voice Input for Interactive Control. In: ACM UIST 2001, pp. 155-156. ACM Press, New York (2001)

5. Iwata, H.: Art and Technology in Interface Devices. In: ACM VRST 2005, pp. 1-7. ACM Press, New York (2005)

6. Kawamura, H., Kadota, H., Yamamoto, M., Takaya, T., Ohuchi, A.: Development of an Entertainment Indoor Blimp Robot Based On Hovering Control. SOFT 17, 2, 203-211 (2004) (in Japanese)

7. Knowbotic Research: Naked Bandit/here, not here/white sovereign, http: / / www.krcf.org/

8. Lightship, http: //www. Iightships.com/

9. Marvin, C.: When Old Technologies Were New. Oxford University Press, UK (1988)

10. Passino, k.M., Yurkovich, S.: Fuzzy Control. Prentice Hall, Englewood Cliffs (1998)

11. Paulos, E., Canny, J.: PRoP: Personal Roving Presence. In: ACM CHI 1998, pp. 296-303. ACM Press, New York (1998)

12. Reeves, S., Benford, S., O'Malley, C., Fraser, M.: Designing the Spectator Experience. In: ACM CHI 2005, pp. 741-750. ACM Press, New York (2005)

13. Yoshimoto, H., Hori, K.: Design of Blimps for Interactive Media and Arts. In: MAST Workshop, http: / /mast.mat.ucsb.edu/

14. Yoshimoto, H., Jo, K., Hori, K.: Design of Installation with Interactive UAVs. In: ACM ACE 2008, p. 424. ACM Press, New York (2008) 